\title{
Arylazo-1,2,3-triazoles: "clicked" photoswitches for versatile functionalization and electronic decoupling
}

Dong Fang, Zhao-Yang Zhang,* and Tao Li*

School of Chemistry and Chemical Engineering, Frontiers Science Center for Transformative Molecules, Shanghai Key Laboratory of Electrical Insulation and Thermal Aging, Shanghai Jiao Tong University, Shanghai 200240, China

*Email: zy_zhang@sjtu.edu.cn; litao1983@sjtu.edu.cn

\begin{abstract}
The development of light-responsive chemical systems often relies on the rational design and suitable incorporation of molecular photoswitches such as azobenzenes. Linking a photoswitch core with another $\pi$-conjugated molecular entity may give rise to intramolecular electronic coupling, which can dramatically impair the photoswitch function. Decoupling strategies have been developed based on additionally inserting a linker that can disrupt the through-bond electronic communication. Here we show that 1,2,3-triazole — a commonly used decoupling spacer — can be directly merged into the azoswitch core to construct a class of "self-decoupling" azoswitches arylazo-1,2,3-triazoles. These heteroaryl azoswitches are easily accessed and readily functionalized using click chemistry. Their photoswitch property can be regulated by structural modification, enabling (near-)quantitative $E-Z$ photoconversion and widely tunable $Z$-isomer thermal half-lives from days to years. Combined experimental and theoretical results demonstrate that the electronic structure of the photoswitch core is not substantially affected by various substituents attached to the 1,2,3-triazole unit, benefitting from its cross-conjugated nature. The combination of clickable synthesis, tunable photoswitch property and self-decoupling ability, makes arylazo-1,2,3-triazoles intriguing molecular tools in designing photo-responsive systems with desired performance.
\end{abstract}




\section{Introduction}

Molecular photoswitches have become important tools for remote control over the structure, property, and function of a system using light irradiations, ${ }^{1-5}$ which has received widespread attention in smart materials, ${ }^{6-9}$ optoelectronic devices, ${ }^{10-13}$ pharmacology and biomedicine, ${ }^{14-17}$ solar energy storage and conversion, ${ }^{18-22}$ etc. Azobenzenes are a prevailing class of photoswitches, benefitting from their simple chromophore structure and robust $E \leftrightarrows Z$ photoisomerizations that induce large geometrical changes. ${ }^{23-26}$

There are a plethora of azobenzene derivatives obtained by introducing substituents to phenyl rings. In order to further expand the molecular diversity and tune the photoswitch properties, an increasing interest has been recently focused on developing heteroaryl azo molecules, also refered to as azoheteroarenes, by replacing the phenyl ring with a heteroaromatic ring. ${ }^{27}$ Heteroaryl azoswitches can be tailor-made by selecting a specific heteroaryl group characterized by ring size, type/number/position of the heteroatom, and its linking position to azo group, all having significant influence on the geometric/electronic structure of $E$ and $Z$ isomers. Of particular interest are azoheteroarenes bearing five-membered heterocycles such as imidazole, ${ }^{28-31}$ pyrazole, $^{32-38}$ and thiophene. ${ }^{39-41}$ Phenylazoimidazoles, $^{29}$ phenylazopyrazoles ${ }^{32-33}$ and phenylazothiophenes ${ }^{39-40}$ could display an unusual T-shaped geometry for their $Z$-isomers (where phenyl ring is perpendicular to the heterocycle), which is not accessible by $Z$-azobenzenes due to the steric hindrance between two six-membered rings. Significantly, the T-shaped $Z$-isomers of phenylazopyrazoles, stabilized by $\mathrm{C}-\mathrm{H} \cdots \pi$ interaction, possessed extremely high thermal stability with half-life $\left(t_{1 / 2}\right)$ up to 1000 days. ${ }^{32-33}$ Nonetheless, the T-shaped $Z$-phenylazothiophenes, stabilized by unique lone-pair $\cdots \pi$ interaction, showed $t_{1 / 2}$ of only a few hours. ${ }^{39-40}$ These phenylazoheteroarenes generally offered quantitative $E \rightarrow Z$ photoisomerization, but the reverse photoconversion could be suppressed due to low $n-\pi^{*}$ absorbance of the T-shaped $Z$-isomers. Several azobisheteroarenes were also reported, including azobisimidazoles, ${ }^{31}$ azobisthiophenes ${ }^{41}$ and azobispyrazoles, ${ }^{42}$ which generally exhibited twisted $Z$-isomers that facilitated their $Z \rightarrow E$ photoisomerization. Significantly, azobispyrazole family showed (near-)quantitative bidirectional photoconversion and their $Z$-isomer $t_{1 / 2}$ was broadly tunable from hours to years. ${ }^{42}$ These advances demonstrate the distinct characteristics of various azoheteroarenes brought by different heteroaryl groups and the great potential of heteroaryl motif in tuning the structure and property of azo photoswitches.

As a building block for light control applications, a molecular photoswitch generally needs to be linked with other molecular entities or integrated into a more complex system. A major consideration is whether the intrinsic photoswitch properties can be preserved after incorporation. It is well-documented that the photoreactivity can be dramatically decreased if a photoswitch is electronically coupled to another $\pi$-conjugated unit. ${ }^{43-49}$ To address this issue, the effect of linker structure on the strength of electronic coupling has been widely investigated, and several decoupling strategies for azobenzenes have been developed, as illustrated in Figure 1a. Complete decoupling can be simply achieved by inserting a sp ${ }^{3}-\mathrm{CH}$ group as the spacer, e.g., a methylene or an aliphatic chain, which is nonetheless not suitable for designing rigid molecular architectures. In a rigid and linear architecture where an azobenzene unit is merged with a 
biphenyl bridge, the electronic coupling strength can be greatly attenuated by increasing the torsion angle between two phenyl planes to $\sim 90^{\circ} .50-52$ In quest of efficient multiphotochromic molecules, ${ }^{53}$ bis/tris-azobenzenes were developed where two/three azobenzene units shared the central benzene ring via ortho-, meta-, or para-connections. ${ }^{54-60}$ The extended $\pi$-conjugation in ortho- and para-compounds led to severe reduction (or complete loss) of the photoisomerization ability and Z-isomer stability, while the cross-conjugation effect ${ }^{61}$ of meta-connection suppressed the electronic communication between azo-photochromophores and they could behave similarly to individual azobenzenes. ${ }^{54-60}$ An alternative approach to cross-conjugation is employing 1,2,3-triazole ring as the linker group, which can disrupt the through-bond electronic communication between 1- and 4- substituents, ${ }^{62}$ and such structure can be readily accessed by click chemistry, i.e., $\mathrm{Cu}(\mathrm{I})$-catalyzed azide-alkyne cycloaddition, CuAAC. ${ }^{63-64}$ Barrett group "clicked" diverse end groups to azobenzene chromophores and found that, these derivatives preserved the photophysical characteristics of the pristine chromophores and their $Z$-isomer $t_{1 / 2}$ were retained within a single order of magnitude. ${ }^{65-66}$ Amar et al. connected azobenzene unit to bipyridine ligand through 1,2,3-triazole, and their combined experimental and computational study showed that the electronic structure and photophysical properties of azobenzene remained almost unchanged after coordination to metal cations. ${ }^{67}$

a)
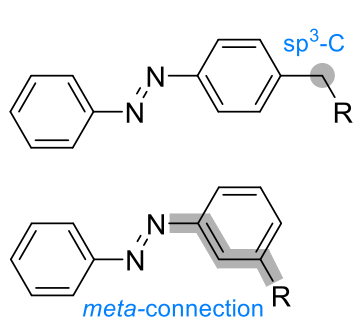

c)

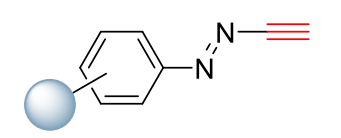

Decoupling strategies

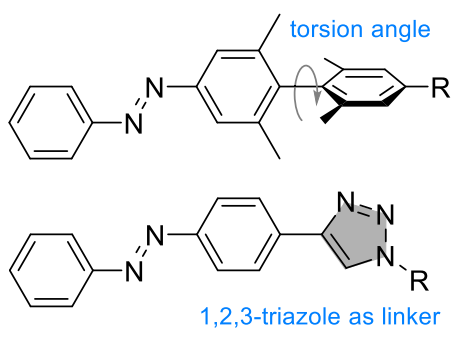

$+\mathrm{N}_{3}-\mathrm{\longrightarrow} \stackrel{\text { "Click" }}{\longrightarrow}$ b) This work:
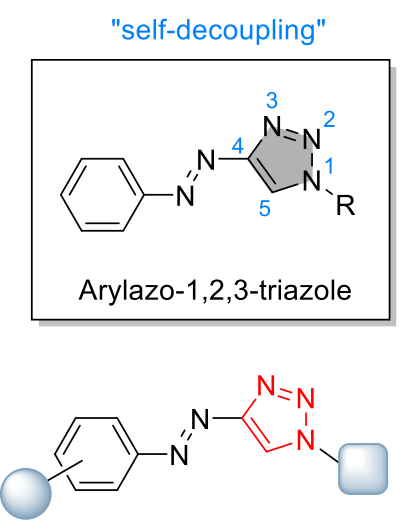

Figure 1. a) Typical strategies for decoupling of azobenzene unit. b) Arylazo-1,2,3-triazole as a "self-decoupling" azoswitch developed in this work. c) A schematic representation of the "click" approach to arylazo-1,2,3-triazoles.

We envision that 1,2,3-triazole, as a unique heteroaryl group, can be directly bonded to azo group to construct "self-decoupling" heteroaryl azoswitches. Therefore, we are motivated to investigate the synthesis, structure, and photoswitch property of arylazo-1,2,3-triazoles, as shown in Figure 1b. Such azoswitches can be easily accessed and readily functionalized by virtue of click chemistry (Figure 1c). We believe that arylazo-1,2,3-triazoles are interesting and useful azoswitches for the following reasons: 1) they enable straightforward electronic decoupling of the photochromic core; 2) they may show excellent photoswitch properties like other heteroaryl azo molecules; 3 ) they could be versatile building blocks for a 
wide array of applications, benefitting from the multifunctionality of 1,2,3-triazole, ${ }^{62,68-70} \mathrm{e.g}$,. ligands to metal ions, ${ }^{71}$ H-bond donors/acceptors, ${ }^{72}$ anion receptors, ${ }^{73}$ anchoring groups on metal surfaces, ${ }^{74}$ linkers promoting intramolecular charge transfer. ${ }^{75}$

\section{Results and discussion}

\section{Synthesis}

Taking advantage of the orthogonality of click reaction, we utilized a convenient one-pot approach to arylazo-1,2,3-triazoles where the formation of azide/alkyne and their cycloaddition could take place in the same reaction mixture, as shown in Scheme 1 (see Supporting Information for experimental details). This could also avoid the explosion risk of organic azides. Arylazoethynyl-Tips (AAET), the precursor of arylazoacetylene, were readily obtained via coupling between aryldiazonium tetrafluoroborate and lithiumethynyl-Tips in yields of $82 \%-91 \%$. The one-pot click reaction proceeded by 1 ) in situ formation of alkyl and aryl azides at first, respectively by substitution reaction between alkyl halides and $\mathrm{NaN}_{3}$ and diazotization-azidation of arylamines in the presence of $t$-BuONO and $\mathrm{TMSN}_{3}$, and 2) deprotection of AAET upon addition of tetra- $n$-butylammonium fluoride (TABF) to give the arylazoacetylenes, and 3) immediate cycloaddition between alkyl/aryl azides and arylazoacetylenes under catalysis of $\mathrm{Cu}^{+}$(which was pre-formed in the mixture by reduction of $\mathrm{CuSO}_{4}$ with sodium ascorbate). A representative family of 17 arylazo-1,2,3-triazoles were synthesized in yields of $37 \%$ to $93 \%$.

Because rich kinds of aromatic amines and organic halides are commercially available, a broad variety of AAET and organic azides are readily accessed. This enables the construction of arylazo-1,2,3-triazoles in a modular fashion that can be functionalized with a vast scope of substituents at both sides.

Scheme 1. Synthesis of arylazo-1,2,3-triazoles.

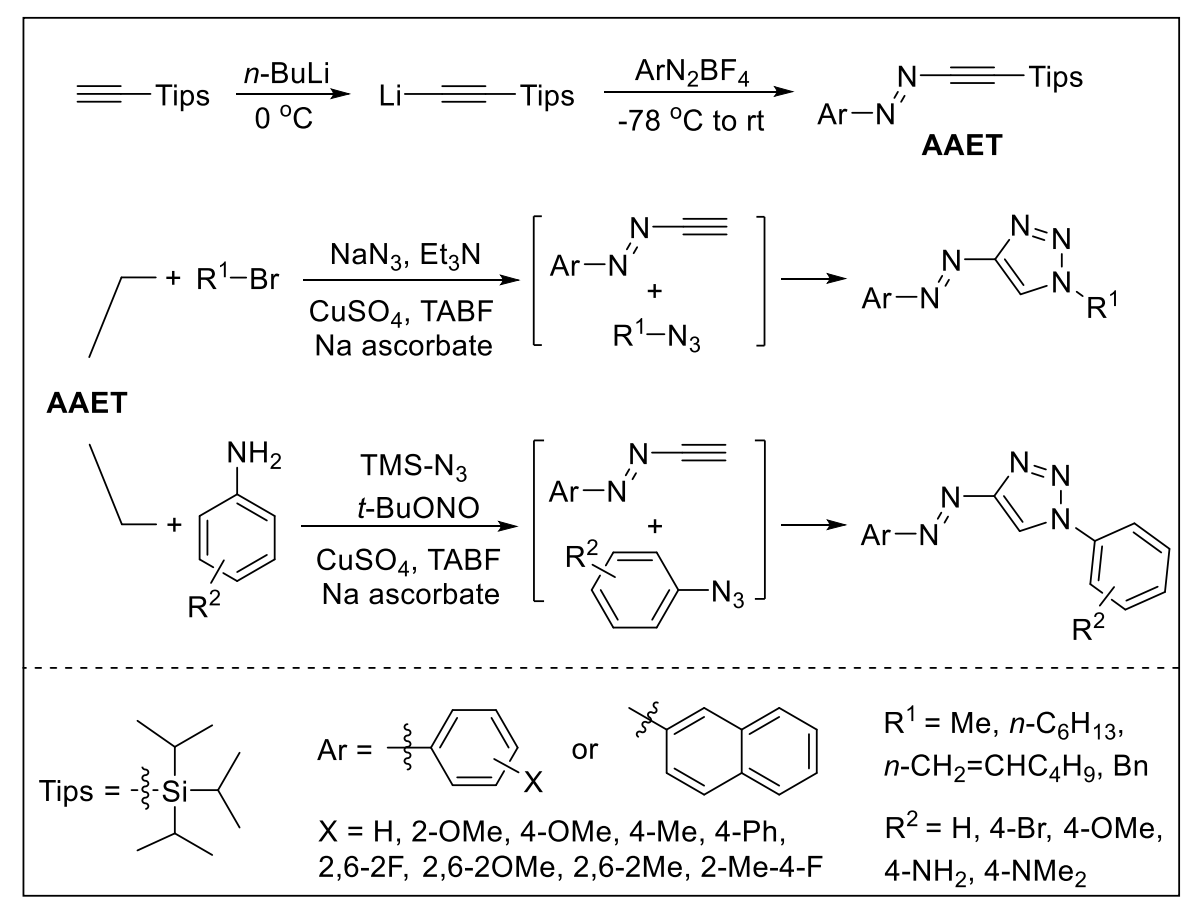




\section{Structure and photoswitch properties}

We first investigated the structure and photoswitch properties of phenylazo-1,2,3-triazole $\mathbf{1}$, the simplest and parent molecule in this family, with a combination of experimental results and quantum chemical calculations. Photoisomerization properties were studied using UV-Vis absorption spectra in DMSO (Figures 2 and S3). E-1 showed a strong $\pi-\pi^{*}$ absorption band with $\lambda_{\max }$ of $329 \mathrm{~nm}$ and a very weak $\mathrm{n}-\pi^{*}$ band at $425 \mathrm{~nm}$ (Figure 2a and Table S1). Excitation of the $\pi-\pi^{*}$ transition using $350 \mathrm{~nm}$ light led to almost complete $E \rightarrow Z$ photoisomerization in $96 \%$ yield. The $Z$ isomer had a slightly stronger $\mathrm{n}-\pi^{*}$ band with $\lambda_{\max }$ of $422 \mathrm{~nm}$. Due to the heavy overlapping of the two $\mathrm{n}-\pi^{*}$ bands with $\varepsilon(Z) / \varepsilon(E)$ ratio only $\sim 1.5$ at most (Figure 2a inset), selective excitation of the $Z$ isomer was impossible. Therefore, $Z \rightarrow E$ back photoconversion was not effective and the highest $E$ isomer content in PSS was only about $65 \%$ at an optimized irradiation wavelength $(400 \mathrm{~nm})$. The thermal $Z \rightarrow E$ isomerization kinetics (first-order) at elevated temperatures were measured in DMSO (Figure S8 and Table S2), which revealed a quite long thermal $t_{1 / 2}$ of $105 \mathrm{~d}$ at $25^{\circ} \mathrm{C}$.
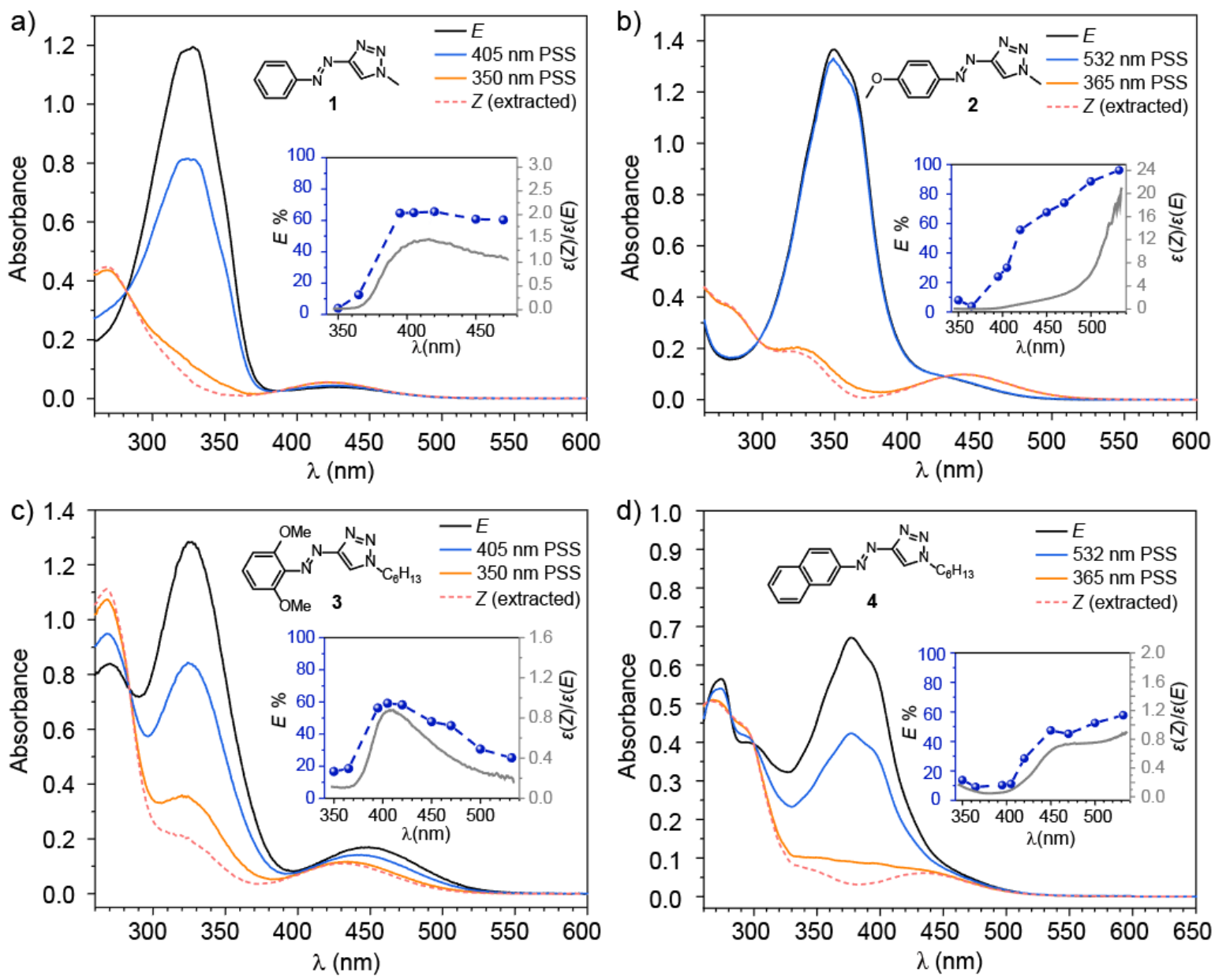

Figure 2. UV-Vis absorption spectra of arylazo-1,2,3-triazoles 1-4 in DMSO. Insets show E-isomer fractions at various PSSs and $Z / E$ absorptivity ratio as a function of wavelength. 
Table 1. Spectroscopic data, photoconversions, and $Z$-isomer $t_{1 / 2}$ of 1-4 measured in DMSO.

\begin{tabular}{|c|c|c|c|c|c|c|}
\hline & \multicolumn{2}{|c|}{$E$-isomer } & \multirow{2}{*}{$\frac{Z \text {-isomer }}{\mathrm{n}-\pi^{*} \lambda_{\max }}$} & \multicolumn{2}{|c|}{ Photoconversion $^{[b]}$} & \multirow{3}{*}{$\begin{array}{c}t_{1 / 2} \\
\text { at } 25^{\circ} \mathrm{C} \\
\text { (d) }\end{array}$} \\
\hline & $\pi-\pi^{*} \lambda_{\max }$ & $\mathrm{n}-\pi^{*} \lambda_{\max }$ & & $E \rightarrow Z$ & $Z \rightarrow E$ & \\
\hline & $(\mathrm{nm})$ & $(\mathrm{nm})$ & $(\mathrm{nm})$ & $(\%)$ & $(\%)$ & \\
\hline 1 & 328 & 425 & 422 & $96^{[\mathrm{c}]}$ & $65^{[\mathrm{e}]}$ & $105^{[\mathrm{g}]}$ \\
\hline 2 & 349 & $-[\mathrm{a}]$ & 439 & $96^{[\mathrm{d}]}$ & $96^{[\mathrm{f}]}$ & $17^{[\mathrm{g}]}$ \\
\hline 3 & 327 & 449 & 432 & $83^{[c]}$ & $59^{[\mathrm{e}]}$ & $717^{[\mathrm{g}]}$ \\
\hline 4 & 378 & $-[a]$ & 434 & $91^{[\mathrm{d}]}$ & $58^{[\mathrm{f}]}$ & $2.5^{[\mathrm{h}]}$ \\
\hline
\end{tabular}

[a] The $n-\pi^{*}$ peak position cannot be distinguished due to overlapping with the tail of $\pi-\pi^{*}$ band. [b] The isomer contents were obtained from integration results of ${ }^{1} \mathrm{H}$ NMR spectra in DMSO- $d_{6}$ (see Figures S4-S7). [c]-[f] The samples were irradiated by lights of [c] $350 \mathrm{~nm}$, [d] $365 \mathrm{~nm}$, [e] $400 \mathrm{~nm}$, and [f] 532 nm. [g] Values at $25{ }^{\circ} \mathrm{C}$ were extrapolated from kinetics data at elevated temperatures using Arrhenius equation (see Section 2.2 in SI). [h] Value was measured at $25^{\circ} \mathrm{C}$ directly.

Both isomers have two stable ground state conformations (due to the rotation of triazole ring relative to phenylazo group), which were optimized using density functional theory (DFT), and their electronic transitions were computed with time-dependent DFT (TDDFT). For $E$-1, its two conformers $E-1(1)$ and $E$-1(2) showed the commonly found planar geometry, as shown in Figure 3a. E-1(1) is more favorable with a population of $98.2 \%$ (Boltzmann distribution at $25{ }^{\circ} \mathrm{C}$ in DMSO, see Table S5). The higher energy of $E-1$ (2) could be attributed to the repulsive interaction between two nitrogen lone pairs (from an azo nitrogen and N3 on triazole, which oriented towards each other), as illustrated in Figure 3a. Such lone-pair $\cdots$ lone-pair repulsion was confirmed by i) a repulsive N...N noncovalent interaction (NCI) surface (Figures 3a and S12) and ii) obvious polarization of the involved $\mathrm{N}$ lone pairs visualized by the electron localization function (ELF) map (Figures 3a and S13). For Z-1, the dominant conformer Z-1(2) adopted a nearly perfect $\mathrm{T}$-shaped geometry $\left(\varphi_{\mathrm{CCNN}}=91.6^{\circ}\right)$ stabilized by a favorable $\mathrm{C}-\mathrm{H}^{\cdots} \cdots \pi$ interaction (visualized by NCI surface and ELF map), as depicted in Figure 3b, while the twisted conformer Z-1(1) was less favorable. Such T conformation, on the one hand, led to weak $\mathrm{n}-\pi^{*}$ absorbance (oscillator strength $f$ of only 0.0021 ) and thus disfavored the $Z \rightarrow E$ photoconversion, and, on the other hand, underlay the long thermal $t_{1 / 2}$ of Z-1. This is consistent with the structure-property relationship established by Fuchter et al for other $\mathrm{N}$-heterocycle based azoheteroarenes. ${ }^{33}$ 

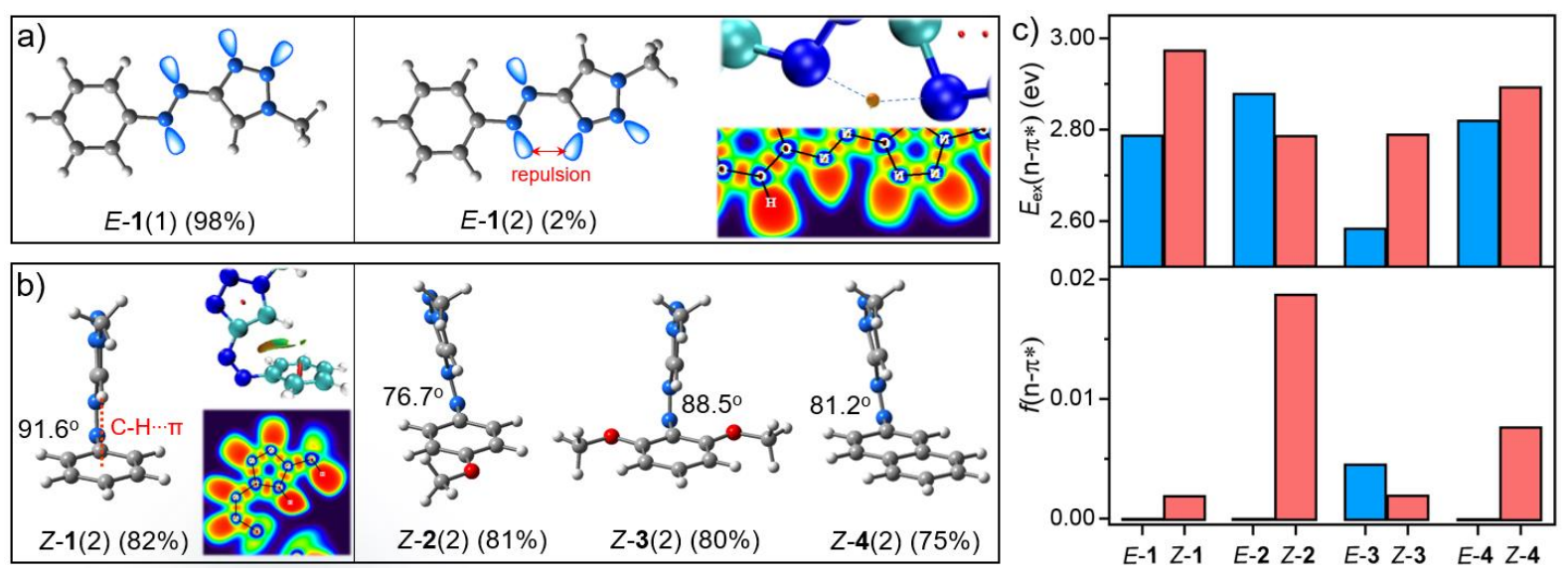

Figure 3. a) Geometry of two conformers of $E-1$ with a representation of nitrogen lone pairs (Boltzmann distributions at $25{ }^{\circ} \mathrm{C}$ are shown in parentheses). The right side shows a NCI surface and an ELF map that describes lone-pair ' lone-pair repulsion between two $\mathrm{N}$ atoms in $E-\mathbf{1}$ (2). b) Geometry of the dominant $Z$ conformers of 1-4. A NCI surface and an ELF map that describes C-H $\cdots \pi$ interaction in Z-1 (2) are also shown. The geometry was optimized at the PBE0-D3(BJ)/6-311G**/SMD (DMSO) level of theory. c) Excitation energy and oscillator strength of $n-\pi^{*}$ transitions calculated at the TD-PBE0/6-311G**/SMD (DMSO) level of theory.

The structure and photoswitch properties were then tuned by structural modifications at benzene ring. As show in Figure 2b, para-OMe-substituted molecule 2 showed a $\pi-\pi^{*} \lambda_{\max }$ of $349 \mathrm{~nm}$, which were redshifted by $20 \mathrm{~nm}$ relative to 1 . This resulted from the $\mathrm{p}-\pi$ conjugation effect that effectively reduced the energy gap between HOMO ( $\pi$ orbital) and LUMO ( $\pi^{*}$ orbital), as verified by theoretical computations (Table S6). $96 \%$ yield of $E \rightarrow Z$ isomerization reached by $365 \mathrm{~nm}$ light irradiation. Moreover, the $\mathrm{n}-\pi^{*}$ bands of $E-\mathbf{2}$ and Z-2 were well separated and a high $\varepsilon(Z) / \varepsilon(E)$ ratio above 20 was obtained at $532 \mathrm{~nm}$ (Figure $2 \mathrm{~b}$ inset), which enabled $96 \%$ yield of $Z \rightarrow E$ isomerization under $532 \mathrm{~nm}$ light. Such effective back photoconversion benefitted from the ability of para-OMe group to 1) simultaneously increase the n- $\pi^{*}$ transition excitation energy of $E$ isomer and decrease that of $Z$ isomer (Figure 3c), i.e, blue-shift the $E$-isomer $\mathrm{n}-\pi^{*}$ band and red-shift the $Z$-isomer $\mathrm{n}-\pi^{*}$ band, and 2) effectively twist the shape of $Z$-isomer $\left(\varphi_{\mathrm{CCNN}}=76.7^{\circ}\right.$, see Figure $\left.3 b\right)$ and thus remarkably enhance $Z$-isomer $n-\pi^{*}$ absorbance $(f$ was increased by one order of magnitude, see Figure $3 \mathrm{c}$ and Table S6). The twisted Z-isomer geometry in turn led to a shortened $t_{1 / 2}$ of $17 \mathrm{~d}$. These substitution effects of para-OMe were also observed for phenylazopyrazoles in previous work. ${ }^{34,36,38}$

We then introduced two methoxy groups to ortho-positions (molecule 3, see Figure 2c). Due to the repulsive interaction between methoxy and azo group, ${ }^{76}$ the $E$-isomer adopt a nonplanar conformation where phenyl ring was rotated relative to the azopyrazolyl part $\left(\varphi_{\mathrm{CCNN}}=8.2^{\circ}\right)$. This weakened the $\pi$-conjugation and blue-shifted the $\pi-\pi^{*} \lambda_{\max }$ by $4 \mathrm{~nm}$ relative to $E$-1. Distorted conformation also made the $\mathrm{n}-\pi^{*}$ transition (which was symmetry-forbidden) more allowed, and thus the $f$ was improved to 0.0045 
(perfect zero for planar $E$-isomers). On the other hand, Z-3(2) adopted a nearly T shaped conformation $\left(\varphi_{\mathrm{CCNN}}=88.5^{\circ}\right)$, resulting in a less allowed $\mathrm{n}-\pi^{*}$ transition with $f$ of only 0.0019 . Therefore, we observed the lower absorbance of $\boldsymbol{Z - 3}$ than $E-\mathbf{3}$ throughout the n- $\pi^{*}$ region (Figure 2c). Naturally, effective $Z \rightarrow E$ photoisomerization was inhibited, and the highest $E$-isomer content of about $60 \%$ was obtained at the PSS of $400 \mathrm{~nm}$, where $\varepsilon(Z) / \varepsilon(E)$ ratio reached a maximum value of about 0.9 (Figure $2 \mathrm{c}$ inset). On the other hand, $E \rightarrow Z$ isomerization could be induced by visible lights taking advantage of the higher $n-\pi *$ absorbance of $E-\mathbf{3}$ over $Z-\mathbf{3}$, and $74.8 \%$ photoconversion was found under $532 \mathrm{~nm}$ light $(\varepsilon(Z) / \varepsilon(E)$ ratio $\sim 2.5$ ). Notably, 3 provided an extremely long-lived Z-isomer with $t_{1 / 2}$ of $717 \mathrm{~d}$, benefitting from the $\mathrm{T}$ shape and bis-ortho-OMe effects that were observed in previous work. ${ }^{77}$

The replacement of benzene with naphthalene (molecule 4) strongly redshifted the $\pi-\pi^{*} \lambda_{\max }$ of $E$-isomer to $378 \mathrm{~nm}$ (Figure 2d). As a result, $405 \mathrm{~nm}$ visible light induced effective $E \rightarrow Z$ isomerization with $89 \%$ yield. The red-shifted $\pi-\pi^{*}$ band, on the other side, left a strong tail absorbance above $450 \mathrm{~nm}$ that was higher than the $\mathrm{n}-\pi^{*}$ absorbance of $Z$-isomer, which disfavored the $Z \rightarrow E$ photoconversion (only $58 \%$ of $E$-isomer at $532 \mathrm{~nm}$ PSS). Meanwhile, the $Z$-isomer $t_{1 / 2}$ become 42 times shorter ( $2.5 \mathrm{~d}$ for Z-4 vs. $105 \mathrm{~d}$ for Z-1), which was also associated with the extension of the conjugated system. Interestingly, a near T-shaped conformation $\left(\varphi_{\mathrm{CCNN}}=81.2^{\circ}\right)$ was theoretically found for Z-4 (Figure 3b), although it showed such a short $t_{1 / 2}$.

In brief, structural modification on the benzene ring side allowed reformation of the electronic and geometric structures of arylazo-1,2,3-triazoles. $E \rightarrow Z$ photoisomerization yields were always excellent, and $Z$-isomer $t_{1 / 2}$ were tuned from days to years, while the $Z \rightarrow E$ photoconversion was disfavored by $T$ shaped geometry of $Z$-isomer. Photoswitch 2 with para-OMe provided the best compromise between the two aspects, enabling $96 \%$ photoconversion in both directions and a fairly long $t_{1 / 2}$ of $17 \mathrm{~d}$.

\section{Electronic decoupling}

In the following, we investigated the ability of 1,2,3-triazole ring to decouple the photoswitching core from its substituent groups. Molecule $\mathbf{2}$ was used as a parent arylazotriazole photoswitch, and a series of derivatives $\mathbf{2} \mathbf{P h}-\mathbf{X}$ were studied, where a phenyl ring was linked to 1,2,3-triazole to extend $\pi$ conjugation and different end groups $\left(\mathrm{X}=\mathrm{H}, \mathrm{Br}, \mathrm{OMe}, \mathrm{NH}_{2}\right.$, and $\left.\mathrm{NMe}_{2}\right)$ were introduced to para-position to induce different electronic effects. Azobenzene analogues $\mathbf{5}$ and $\mathbf{5 P h}-\mathbf{X}$ were taken as reference compounds.

UV-Vis spectra in Figure 4a clearly demonstrated the significant decoupling effect of 1,2,3-triazole unit. For the azobenzene series, the $\pi-\pi^{*}$ absorption bands displayed a large red-shift of $16 \mathrm{~nm}\left(\lambda_{\max }\right.$ from 344 $\mathrm{nm}$ to $360 \mathrm{~nm}$ ) when the conjugated system was extended from $\mathbf{5}$ to $\mathbf{5 P h}-\mathbf{H}$, and increasing the electron richness of end groups progressively shifted the $\lambda_{\max }$ of $\mathbf{5 P h}-\mathbf{X}$ from $360 \mathrm{~nm}$ to $402 \mathrm{~nm}$. In contrast, arylazotriazole $\mathbf{2} \mathbf{P h}-\mathbf{H}$ showed a small red-shift of $6 \mathrm{~nm}$ relative to 2 (from $344 \mathrm{~nm}$ to $350 \mathrm{~nm}$ ). More significantly, the $\lambda_{\max }$ of $\mathbf{2 P h}-\mathbf{X}$ remained almost unchanged with different end groups, even neglecting the strong electron-donation effect of $-\mathrm{NMe}_{2}$. These results indicated that the effective 
electronic conjugation commonly seen in azobenzene-based systems was largely weakened by the arylazo-1,2,3-triazole chromophore.

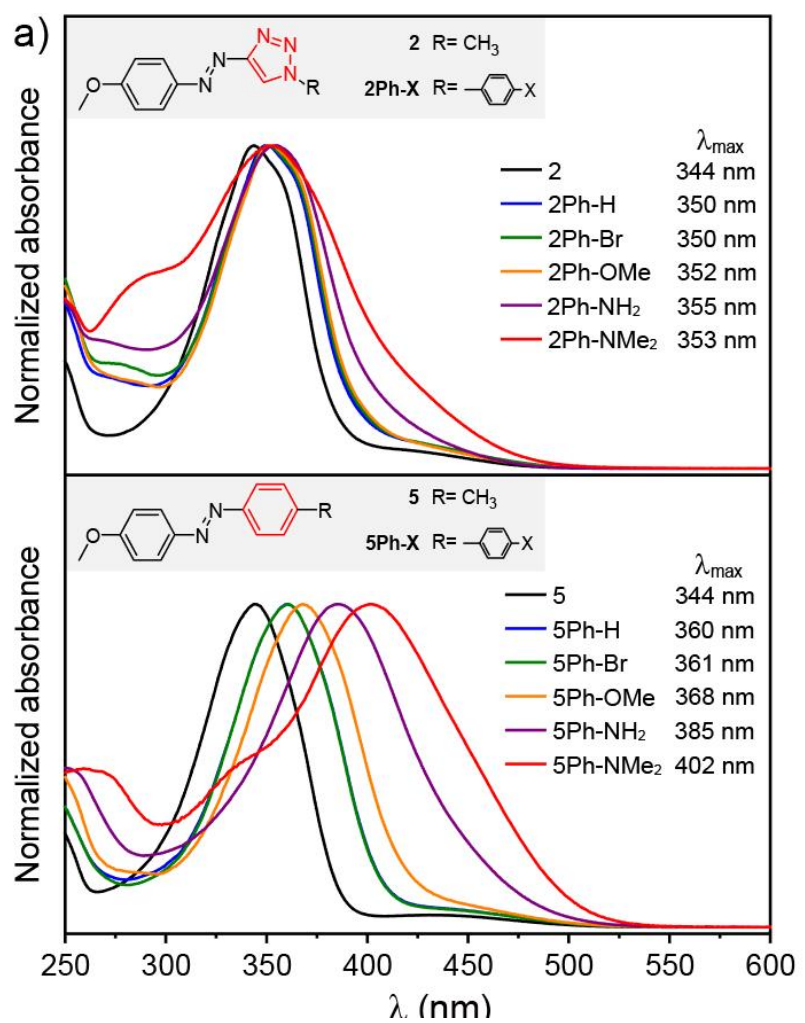

b) $L+1$

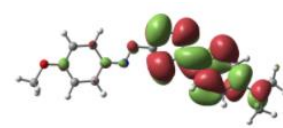

L

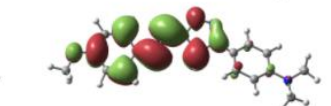

$\mathrm{H}$

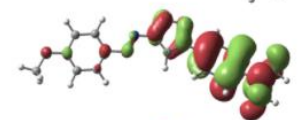

$\mathrm{H}-1$

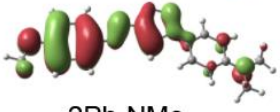

2Ph-NMe cross-conjugation

c)

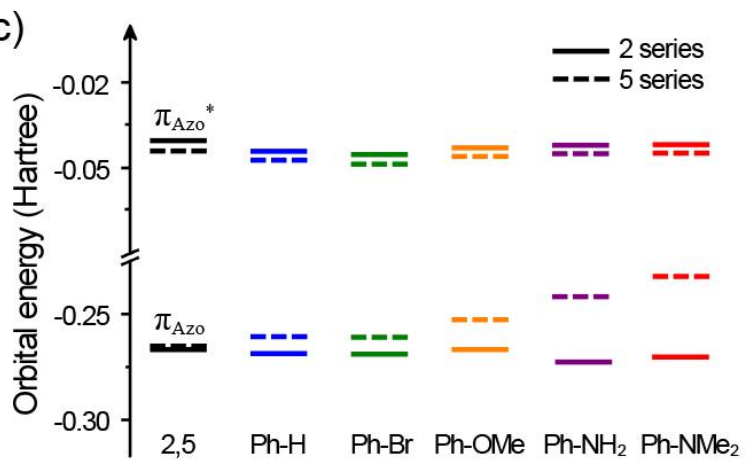

Figure 4. a) UV-Vis absorption spectra of 2-based arylazo-1,2,3-triazole derivatives and their azobenzene analogues in MeCN with a list of their $\pi-\pi^{*} \lambda_{\max }$. The shoulders at 250-300 $\mathrm{nm}(300-350 \mathrm{~nm})$ in the spectra of $\mathbf{2} \mathbf{P h}-\mathrm{NMe}_{2}$ and $\mathbf{5 P h}-\mathrm{NMe}_{2}$ come from electronic transition of the Ph-NMe $\mathrm{N}_{2}$ moiety. b) Isosurface plots of frontier molecular orbitals of $\mathbf{2} \mathbf{P h}-\mathbf{N M e}_{2}$ and $\mathbf{5 P h}-\mathbf{N M e}_{2}$ (calculated at the TD-CAM-B3LYP/6-311G**/SMD (MeCN) level of theory). c) Energy levels of $\pi_{\mathrm{Azo}}$ and $\pi_{\mathrm{Azo}} *$ orbitals for the two series of molecules (see their orbital plots in Figure S14).

To rationalize the experimental findings, we examined the relevant frontier orbitals involved in $\pi-\pi^{*}$ transitions, i.e., the two highest-lying $\pi$ orbitals (HOMO and HOMO-1) and two lowest-lying $\pi^{*}$ orbitals (LUMO and LUMO+1), which were depicted in Figure $3 \mathrm{~b}$. As expected, the $\pi$ and $\pi^{*}$ orbitals of azobenzenes $\mathbf{5 P h}-\mathbf{X}$ were delocalized widely over the entire molecular backbone. In the case of arylazotriazoles $\mathbf{2} \mathbf{P h}-\mathbf{X}$, however, the long-range electronic delocalization was almost cut off, resulting in two electronically decoupled subsystems. Specifically, the $\pi$ and $\pi^{*}$ orbitals of lower energy levels (HOMO-1 and LUMO) were predominantly located on the arylazotriazole part, which is the photochromic core, with a very weak participation of the Ph-X moiety. Meanwhile, the higher-energy-level $\pi$ and $\pi^{*}$ orbitals (HOMO and LUMO+1) were centered on the triazole-Ph-X part, with little wavefunction extension to the arylazo moiety. Such spatial separation characteristic of $\pi$ and $\pi^{*}$ orbitals manifested the cross-conjugated nature of 1,2,3-triazole unit in arylazo-1,2,3-triazole photoswitches. 
In addition to the qualitative orbital picture, orbital energy was further analyzed. It is known that electronic coupling of two units results in energy splitting of the involved orbitals, characterized by increase/decrease of the orbital energy relative to that of the independent units. For the molecules studied here (composed of a photochromic core and a substituent), we focused on $\pi_{\mathrm{Azo}}\left(\pi_{\mathrm{Azo}} *\right)$ orbitals, i.e., the $\pi$ $\left(\pi^{*}\right)$ orbitals distributing on the photochromic core and dominating the lowest $\pi-\pi^{*}$ excited states (see orbital pictures of all molecules in Figure S14). Their orbital energies are shown in Figure $3 \mathrm{c}$. The $\pi_{\mathrm{Azo}} *$ orbitals showed little energy change for both azobenzenes and arylazo-1,2,3-triazoles, but the $\pi_{\mathrm{Azo}}$ orbitals are sensitive to whether electronically decoupled or not. For azobenzenes, the $\pi_{\mathrm{Azo}}$ orbital energy increased by $0.27 \mathrm{eV}$ from $\mathbf{3}$ to $\mathbf{3 P h}-\mathbf{H}$, and energy level of $\mathbf{3 P h}-\mathbf{X}$ continually increased with the electron richness of end groups (by $0.94 \mathrm{eV}$ from $\mathrm{H}$ to $\mathrm{NMe}_{2}$ ), consistent with the trend of $\pi-\pi^{*} \lambda_{\max }$. In contrast, the $\pi_{\mathrm{Azo}}$ orbitals showed a small energy change $(0.075 \mathrm{eV})$ from $\mathbf{2}$ to $\mathbf{2 P h}-\mathbf{H}$, and they basically kept constant with different end groups (with small fluctuations), which supported again the poor electronic coupling between arylazo-1,2,3-triazole core and its substituents.

\section{Conclusion}

In summary, we have developed arylazo-1,2,3-triazoles as heteroaryl azoswitches featuring electronic decoupling characteristic. A convenient route using the CuAAC click reaction is established for facile synthesis and versatile functionalization of these molecules. By structural modifications at the benzene ring side, electronic/geometric structure and thus photoswitch property can be readily tuned. For example, their Z-isomer $t_{1 / 2}$ have been tuned from days to years, and 2 provides $96 \%$ photoconversion in both directions and a fairly long $t_{1 / 2}$ of $17 \mathrm{~d}$. Taking 2 as a parent molecule, we show that the cross-conjugated nature of 1,2,3-triazole unit largely disrupt the electronic communication between the photoswitch core and its substituents. The combination of clickable synthesis, tunable photoswitch property and self-decoupling ability, makes arylazo-1,2,3-triazoles intriguing molecular tools in designing photo-responsive systems with desired performance. Although the aryl moieties are limited to benzeneand naphthalene-based groups in current work, many other (hetero)aryl-based azo-1,2,3-triazoles can be envisioned, providing a large scope for structure design, property improvement, and potential applications.

\section{Acknowledgements}

We acknowledge the finical support from the National Key Research and Development Program of China (2017YFA0207500), National Natural Science Foundation of China (22022507, 51973111), Beijing National Laboratory for Molecular Sciences (BNLMS202004) and China Postdoctoral Science Foundation (2020M681279). 


\section{References}

(1) Brieke, C.; Rohrbach, F.; Gottschalk, A.; Mayer, G.; Heckel, A. Light-controlled tools. Angew. Chem. Int. Edit. 2012, 51, 8446-8476.

(2) Gostl, R.; Senf, A.; Hecht, S. Remote-controlling chemical reactions by light: towards chemistry with high spatio-temporal resolution. Chem. Soc. Rev. 2014, 43, 1982-1996.

(3) Qu, D.; Wang, Q.; Zhang, Q.; Ma, X.; Tian, H. Photoresponsive host-guest functional systems. Chem. Rev. 2015, 115, 7543-7588.

(4) Kathan, M.; Hecht, S. Photoswitchable molecules as key ingredients to drive systems away from the global thermodynamic minimum. Chem. Soc. Rev. 2017, 46, 5536-5550.

(5) Baroncini, M.; Silvi, S.; Credi, A. Photo- and redox-driven artificial molecular motors. Chem. Rev. 2020, 120, 200-268.

(6) Theato, P.; Sumerlin, B. S.; Reillyc, R. K. O.; Thomas H. Epps, I. Stimuli responsive materials. Chem. Soc. Rev. 2013, 42, 7055-7056.

(7) Pianowski, Z. L. Recent implementations of molecular photoswitches into smart materials and biological systems. Chem. Eur. J. 2019, 25, 5128-5144.

(8) Nie, H.; Self, J. L.; Kuenstler, A. S.; Hayward, R. C.; Read De Alaniz, J. Multiaddressable photochromic architectures: from molecules to materials. Adv. Opt. Mater. 2019, 1900224.

(9) Goulet Hanssens, A.; Eisenreich, F.; Hecht, S. Enlightening materials with photoswitches. Adv. Mater. 2020, 1905966.

(10) Li, T.; Jevric, M.; Hauptmann, J. R.; Hviid, R.; Wei, Z.; Wang, R.; Reeler, N. E. A.; Thyrhaug, E.; Petersen, S.; Meyer, J. A. S.et al. Ultrathin reduced graphene oxide films as transparent top-contacts for light switchable solid-state molecular junctions. Adv. Mater. 2013, 25, 4164-4170.

(11) Orgiu, E.; Samorì, P. 25th Anniversary article: organic electronics marries photochromism: generation of multifunctional interfaces, materials, and devices. Adv. Mater. 2014, 26, 1827-1845.

(12) Fu, L.; Leng, B.; Li, Y.; Gao, X. Photoresponsive organic field-effect transistors involving photochromic molecules. Chin. Chem. Lett. 2016, 27, 1319-1329.

(13) Huang, X.; Li, T. Recent progress in the development of molecular-scale electronics based on photoswitchable molecules. J. Mater. Chem. C 2020, 8, 821-848.

(14) Velema, W. A.; Szymanski, W.; Feringa, B. L. Photopharmacology: beyond proof of principle. J. Am. Chem. Soc. 2014, 136, 2178-2191.

(15) Broichhagen, J.; Frank, J. A.; Trauner, D. A roadmap to success in photopharmacology. Acc. Chem. Res. 2015, 48, 1947-1960.

(16) Cheng, H.; Yoon, J.; Tian, H. Recent advances in the use of photochromic dyes for photocontrol in biomedicine. Coordin. Chem. Rev. 2018, 372, 66-84. 
(17) Welleman, I. M.; Hoorens, M. W. H.; Feringa, B. L.; Boersma, H. H.; Szymanski, W. Photoresponsive molecular tools for emerging applications of light in medicine. Chem. Sci. 2020, 11, 11672-11691.

(18) Lennartson, A.; Roffey, A.; Moth-Poulsen, K. Designing photoswitches for molecular solar thermal energy storage. Tetrahedron Lett. 2015, 56, 1457-1465.

(19) Dong, L.; Feng, Y.; Wang, L.; Feng, W. Azobenzene-based solar thermal fuels: design, properties, and applications. Chem. Soc. Rev. 2018, 47, 7339-7368.

(20) Sun, C. L.; Wang, C.; Boulatov, R. Applications of photoswitches in the storage of solar energy. ChemPhotoChem 2019, 3, 268-283.

(21) Xiong, Y.; Zhang, L.; Weis, P.; Naumov, P.; Wu, S. A solar actuator based on hydrogen-bonded azopolymers for electricity generation. J. Mater. Chem. A 2018, 6, 3361-3366.

(22) Zhang, Z.; He, Y.; Wang, Z.; Xu, J.; Xie, M.; Tao, P.; Ji, D.; Moth-Poulsen, K.; Li, T. Photochemical phase transitions enable coharvesting of photon energy and ambient heat for energetic molecular solar thermal batteries that upgrade thermal energy. J. Am. Chem. Soc. 2020, 142, 12256-12264.

(23) Yager, K. G.; Barrett, C. J. Novel photo-switching using azobenzene functional materials. J. Photoch. Photobio. A 2006, 182, 250-261.

(24) Bandara, H. M. D.; Burdette, S. C. Photoisomerization in different classes of azobenzene. Chem. Soc. Rev. 2012, 41, 1809-1825.

(25) Baroncini, M.; Ragazzon, G.; Silvi, S.; Venturi, M.; Credi, A. The eternal youth of azobenzene: new photoactive molecular and supramolecular devices. Pure Appl. Chem. 2015, 87, 537-545.

(26) Seki, T. A wide array of photoinduced motions in molecular and macromolecular assemblies at interfaces. B. Chem. Soc. Jpn. 2018, 91, 1026-1057.

(27) Crespi, S.; Simeth, N. A.; Koeinig, B. Heteroaryl azo dyes as molecular photoswitches. Nat. Rev. Chem. 2019, 3, 133-146.

(28) Otsuki, J.; Suwa, K.; Sarker, K. K.; Sinha, C. Photoisomerization and thermal isomerization of arylazoimidazoles. J. Phys. Chem. A 2007, 111, 1403-1409.

(29) Wendler, T.; Schütt, C.; Näther, C.; Herges, R. Photoswitchable azoheterocycles via coupling of lithiated imidazoles with benzenediazonium salts. J. Org. Chem. 2012, 77, 3284-3287.

(30) Schütt, C.; Heitmann, G.; Wendler, T.; Krahwinkel, B.; Herges, R. Design and synthesis of photodissociable ligands based on azoimidazoles for light-driven coordination-induced spin state switching in homogeneous solution. J. Org. Chem. 2015, 81, 1206-1215.

(31) Weston, C. E.; Richardson, R. D.; Fuchter, M. J. Photoswitchable basicity through the use of azoheteroarenes. Chem. Commun. 2016, 52, 4521-4524.

(32) Weston, C. E.; Richardson, R. D.; Haycock, P. R.; White, A. J. P.; Fuchter, M. J. Arylazopyrazoles: azoheteroarene photoswitches offering quantitative isomerization and long thermal half-lives. J. Am. Chem. Soc. 2014, 136, 11878-11881. 
(33) Calbo, J.; Weston, C. E.; White, A. J. P.; Rzepa, H. S.; Contreras-García, J.; Fuchter, M. J. Tuning azoheteroarene photoswitch performance through heteroaryl design. J. Am. Chem. Soc. 2017, 139, 1261-1274.

(34) Devi, S.; Saraswat, M.; Grewal, S.; Venkataramani, S. Evaluation of substituent effect in Z-isomer stability of arylazo-1H-3,5-dimethylpyrazoles: interplay of steric, electronic effects and hydrogen bonding. J. Org. Chem. 2018, 83, 4307-4322.

(35) Stricker, L.; Böckmann, M.; Kirse, T. M.; Doltsinis, N. L.; Ravoo, B. J. Arylazopyrazole Photoswitches in aqueous solution: substituent effects, photophysical properties, and host-guest chemistry. Chem. Eur. J 2018, 24, 8639-8647.

(36) Zhang, Z. Y.; He, Y.; Zhou, Y.; Yu, C.; Han, L.; Li, T. Pyrazolylazophenyl ether-based photoswitches: facile synthesis, (near-)quantitative photoconversion, long thermal half-life, easy functionalization, and versatile applications in light-responsive Systems. Chem. Eur. J 2019, 25, 13402-13410.

(37) Bhunia, S.; Dolai, A.; Samanta, S. Robust bi-directional photoswitching of thiomethyl substituted arylazopyrazoles under visible light. Chem. Commun. 2020, 56, 10247-10250.

(38) Rustler, K.; Nitschke, P.; Zahnbrecher, S.; Zach, J.; Crespi, S.; König, B. Photochromic evaluation of 3(5)-arylazo-1H-pyrazoles. J. Org. Chem. 2020, 85, 4079-4088.

(39) Heindl, A. H.; Wegner, H. A. Rational design of azothiophenes - substitution effects on the switching properties. Chem. Eur. J. 2020, 26, 13730-13737.

(40) Slavov, C.; Yang, C.; Heindl, A. H.; Wegner, H. A.; Dreuw, A.; Wachtveitl, J. Thiophenylazobenzene: an alternative photoisomerization controlled by lone-pair $\cdots \pi$ interaction. Angew. Chem. Int. Edit. 2019, 59, 380-387.

(41) Huddleston, P. R.; Volkov, V. V.; Perry, C. C. The structural and electronic properties of 3,3'-azothiophene photo-switching systems. Phys. Chem. Chem. Phys. 2019, 21, 1344-1353.

(42) He, Y.; Shangguan Z.; Zhang, Z. Y.; Xie, M.; Yu, C.; Li, T. Azobispyrazole family as photoswitches combining (near-) quantitative bidirectional isomerization and widely tunable thermal half-lives from hours to years. Angew. Chem. Int. Edit. 2021. DOI: 10.1002/anie.202103705.

(43) Hunter, C.A. and Sarson, L. D. Azobenzene-porphyrins. Tetrahedron Lett. 1996, 37, 699-702.

(44) Peters, M. V.; Goddard, R.; Hecht, S. Synthesis and characterization of azobenzene-confined porphyrins. J. Org. Chem. 2006, 71, 7846-7849.

(45) Perrier, A.; Maurel, F.; Jacquemin, D. Single molecule multiphotochromism with diarylethenes. Acc. Chem. Res. 2012, 45, 1173-1182.

(46) Castellanos, S.; Grubert, L.; St Er, R.; Hecht, S. Diarylethene photoswitches featuring tetrathiafulvalene-containing aryl units. J. Phys. Chem. C 2013, 117, 23529-23538.

(47) Petersen, A. U.; Broman, S. L.; Olsen, S. T.; Hansen, A. S.; Du, L.; Kadziola, A.; Hansen, T.; Kjaergaard, H. G.; Mikkelsen, K. V.; Brøndsted Nielsen, M. Controlling two-step multimode switching of 
dihydroazulene photoswitches. Chem. Eur. J. 2015, 21, 3968-3977.

(48) Benmensour, M. A.; Ayadi, A.; Akdas-Kilig, H.; Boucekkine, A.; Fillaut, J.; El-Ghayoury, A. Azobased iminopyridine ligands and their rhenium metal complexes: syntheses, spectroscopic, trans-cis photoisomerization and theoretical studies. J. Photoch. Photobio. A 2019, 368, 78-84.

(49) Kunz, A.; Wegner, H. A. 1+1 $\geq 2$ ? Norbornadiene-Azobenzene molecules as multistate photoswitches. ChemSystemsChem 2020. DOI: org/10.1002/syst.202000035.

(50) Bléger, D.; Dokić, J.; Peters, M. V.; Grubert, L.; Saalfrank, P.; Hecht, S. Electronic decoupling approach to quantitative photoswitching in linear multiazobenzene architectures. J. Phys. Chem. B 2011, $115,9930-9940$.

(51) Zhao, F.; Grubert, L.; Hecht, S.; Bléger, D. Orthogonal switching in four-state azobenzene mixed-dimers. Chem. Commun. 2017, 53, 3323-3326.

(52) Schlimm, A.; Löw, R.; Rusch, T.; Röhricht, F.; Strunskus, T.; Tellkamp, T.; Sönnichsen, F.; Manthe, U.; Magnussen, O.; Tuczek, F.et al. Long-distance rate acceleration by bulk gold. Angew. Chem. Int. Edit. 2019, 58, 6574-6578.

(53) Arnaud, F.; Aurelie, P.; Browne, W. R.; Denis, J. Multiphotochromic molecular systems. Chem. Soc. Rev. 2015, 44, 3719-3759.

(54) Cisnetti, F.; Ballardini, R.; Credi, A.; Gandolfi, M. T.; Masiero, S.; Negri, F.; Pieraccini, S.; Spada, G. P. Photochemical and electronic properties of conjugated bis(azo) compounds: an experimental and computational study. Chem. Eur. J. 2004, 10, 2011-2021.

(55) Bellotto, S.; Reuter, R.; Heinis, C.; Wegner, H. A. Synthesis and photochemical properties of oligo-ortho-azobenzenes. J. Org. Chem. 2011, 76, 9826-9834.

(56) Robertus, J.; Reker, S. F.; Pijper, T. C.; Deuzeman, A.; Browne, W. R.; Feringa, B. L. Kinetic analysis of the thermal isomerisation pathways in an asymmetric double azobenzene switch. Phys. Chem. Chem. Phys. 2012, 14, 4374.

(57) Floß, G.; Saalfrank, P. The Photoinduced $E \rightarrow Z$ Isomerization of bisazobenzenes: A surface hopping molecular dynamics study. J. Phys. Chem. A 2015, 119, 5026-5037.

(58) Slavov, C.; Yang, C.; Schweighauser, L.; Boumrifak, C.; Dreuw, A.; Wegner, H. A.; Wachtveitl, J. Connectivity matters - ultrafast isomerization dynamics of bisazobenzene photoswitches. Phys. Chem. Chem. Phys. 2016, 18, 14795-14804.

(59) Yang, C.; Slavov, C.; Wegner, H. A.; Wachtveitl, J.; Dreuw, A. Computational design of a molecular triple photoswitch for wavelength-selective control. Chem. Sci. 2018, 9, 8665-8672.

(60) Galanti, A.; Diez-Cabanes, V.; Santoro, J.; Valášek, M.; Minoia, A.; Mayor, M.; Cornil, J.; Samorì, P. Electronic decoupling in $\mathrm{C} 3$-symmetrical light-responsive tris(azobenzene) scaffolds: self-assembly and multiphotochromism. J. Am. Chem. Soc. 2018, 140, 16062-16070.

(61) Gholami, M.; Tykwinski, R. R. Oligomeric and polymeric systems with a cross-conjugated 
$\pi$-framework. Chem. Rev. 2006, 106, 4997-5027.

(62) Schulze, B.; Schubert, U. S. Beyond click chemistry - supramolecular interactions of 1,2,3-triazoles. Chem. Soc. Rev. 2014, 43, 2522-2571.

(63) Kolb, H. C.; Finn, M. G.; Sharpless, K. B. Click chemistry: diverse chemical function from a few good reactions. Angew. Chem. Int. Edit. 2001, 40, 2004.

(64) Hein, J. E.; Fokin, V. V. Copper-catalyzed azide-alkyne cycloaddition (CuAAC) and beyond: new reactivity of copper(I) acetylides. Chem. Soc. Rev. 2010, 39, 1302-1315.

(65) Goulet-Hanssens, A.; Barrett, C. J. Modular assembly of azo photo-switches using click chemistry allows for predictable photo-behaviour. J. Photoch. Photobio. A 2019, 368, 78-842014, 294, 62-67.

(66) Goulet-Hanssens, A.; Corkery, T. C.; Priimagi, A.; Barrett, C. J. Effect of head group size on the photoswitching applications of azobenzene Disperse Red 1 analogues. J. Mater. Chem. C 2014, 2, 7505-7512.

(67) Amar, A.; Savel, P.; Akdas-Kilig, H.; Katan, C.; Meghezzi, H.; Boucekkine, A.; Malval, J.; Fillaut, J. Photoisomerisation in aminoazobenzene-substituted ruthenium(II) tris(bipyridine) complexes: influence of the conjugation pathway. Chem. Eur. J. 2015, 21, 8262-8270.

(68) Schweinfurth, D.; Hettmanczyk, L.; Suntrup, L.; Sarkar, B. Metal complexes of click-derived triazoles and mesoionic carbenes: electron transfer, photochemistry, magnetic bistability, and catalysis. J. Inorg. Gen. Chem. 2017, 643, 554-584.

(69) Lauko, J.; Kouwer, P. H. J.; Rowan, A. E. 1H-1,2,3-Triazole: From structure to function and catalysis. J. Heterocycl. Chem. 2017, 54, 1677-1699.

(70) Hua, Y.; Flood, A. H. Click chemistry generates privileged $\mathrm{CH}$ hydrogen-bonding triazoles: the latest addition to anion supramolecular chemistry. Chem. Soc. Rev. 2010, 39, 1262-1271.

(71) Mindt, T. L.; Struthers, H.; Brans, L.; Anguelov, T.; Schweinsberg, C.; Maes, V.; Tourwé, D.; Schibli,

R. "Click to helate": Synthesis and installation of metal chelates into biomolecules in a single Step. J. Am. Chem. Soc. 2006, 128, 15096-15097.

(72) Lee, S.; Hua, Y.; Park, H.; Flood, A. H. Intramolecular hydrogen bonds preorganize an aryl-triazole receptor into a crescent for chloride binding. Org. Lett. 2010, 12, 2100-2102.

(73) Liu, Y.; Sengupta, A.; Raghavachari, K.; Flood, A. H. Anion binding in solution: beyond the electrostatic regime. Chem 2017, 3, 411-427.

(74) Li, N.; Zhao, P.; Liu, N.; Echeverria, M.; Moya, S.; Salmon, L.; Ruiz, J.; Astruc, D. "Click” chemistry mildly stabilizes bifunctional gold nanoparticles for sensing and catalysis. Chem. Eur. J. 2014, 20, 8363-8369.

(75) De Miguel, G.; Wielopolski, M.; Schuster, D. I.; Fazio, M. A.; Lee, O. P.; Haley, C. K.; Ortiz, A. L.; Echegoyen, L.; Clark, T.; Guldi, D. M. Triazole Bridges as versatile linkers in electron donor-acceptor conjugates. J. Am. Chem. Soc. 2011, 133, 13036-13054. 
(76) Beharry, A. A.; Sadovski, O.; Woolley, G. A. Azobenzene photoswitching without ultraviolet light. J. Am. Chem. Soc. 2011, 133, 19684-19687.

(77) Calbo, J.; Thawani, A. R.; Gibson, R. S. L.; White, A. J. P.; Fuchter, M. J. A combinatorial approach to improving the performance of azoarene photoswitches. Beilstein J. Org .Chem. 2019, 15, 2753-2764.

(78) Lygo, O. N.; Khodot, E. N.; Ogurtsov, V. A.; Shelaev, I. V.; Gostev, F. E.; Nekipelova, T. D. Dynamics of trans-cis photoisomerization of hetarylazo dyes. High Energy Chem. 2014, 48, 325-329.

(79) Lin, J.; Yang, M.; Qiu, Y.; Chen, W.; Yan, H.; Gao, F.; OuYang, Z.; Dong, W.; Kuang, T. Synthesis, structure, and halo-, photo-, and thermochromism properties of 5-azotriazolyl salicylic acid and its CdII complex. ChemPlusChem 2013, 78, 598-604. 